\title{
Rashba-effect-induced spin dephasing in $n$-typed InAs quantum wells
}

\author{
M. Q. Weng ${ }^{1,2}$ and M. W. $\mathrm{Wu}^{1,2, *}$ \\ ${ }^{1}$ Structure Research Laboratory, University of Science ETechnology of China, Academia Sinica, Hefei, Anhui, 230026, China \\ ${ }^{2}$ Department of Physics, University of Science ETTechnology of China, Hefei, Anhui, 230026, China ${ }^{\dagger}$
}

(Dated: December 7, 2018)

\begin{abstract}
We perform a many-body investigation of the spin dephasing in $n$-typed InAs quantum wells under moderate magnetic fields in the Voigt configuration by constructing and numerically solving the kinetic Bloch equations. We obtain the spin dephasing time due to the Rashba effect together with the spin conserving scattering such as the electron-phonon, the electron-nonmagnetic impurity as well as the electron-electron Coulomb scattering. By varying the initial spin polarization, temperature, impurity density, applied magnetic field and the interface electric field, we are able to study the spin dephasing time under various conditions. For the electron density and quantum well width we study, the many-body effect dominates the spin dephasing. Moreover, we find an anomalous resonance peak in the spin dephasing time for high initial spin polarization under moderate magnetic fields.
\end{abstract}

PACS numbers: 71.10.-w, 67.57.Lm, 72.25.Rb, 73.61.Ey

\section{INTRODUCTION}

Almost all of the current semiconductor devices are based on manipulating electronic charges. The arising field of spintronics proposes to use the spin degree of freedom of electrons in place of/in addition to the charge degree of freedom for device applications in order to add new features and functionalities to semiconductors devices. ${ }^{1,2,3}$ The hope of the realization of the proposed spintronic devices is supported by the resent development of ultrafast nonlinear optical experiments $4,5,6,7,8,9,10,11,12,13,14,15,16,17$ where long spin dephasing time (>100 ns) is reported.

The functionalities of the semiconductor spintronic devices rely on the manipulation of the spin coherence. In order to realize these devices, one needs to thoroughly understand the spin dephasing mechanisms which tend to destroy the spin coherence. Historically, three spin dephasing mechanisms have been proposed in semiconductors: ${ }^{18,19}$ the Elliot-Yafet (EY) mechanism, ${ }^{20,21}$ the D'yakonov-Perel' (DP) mechanism, ${ }^{22}$ and the Bir-Aronov-Pikus (BAP) mechanism. ${ }^{23}$ All of the three mechanisms are either due to the spin flip (SF) scattering or are treated as effective SF scattering. The spin dephasing times of these mechanisms for low polarized system are calculated in the framework of single particle approximation. ${ }^{18}$ In additional to these single particle spin dephasing mechanisms, three years ago $\mathrm{Wu}$ proposed a many-body spin dephasing mechanism which has long been overlooked in the literature. This mechanism is caused by irreversibly disrupting the phases between spin dipoles due to the inhomogeneous broadening together with the spin-conserving (SC) scattering, ${ }^{24,25,26,27,28}$ and is therefore a many-body effect. The inhomogeneous broadening can be introduced by the energy dependence of $g$-factor ${ }^{24,25,29}$ and/or the momentum k-dependence of the DP term. ${ }^{25,26,27}$ Our recent works further show that this mechanism also plays an important role in the spin dephasing during the spin transport. ${ }^{30,31}$

Very recently we performed a systematic investigation $^{32,33}$ of the spin dephasing due to the DP effect in $n$-typed GaAs (100) quantum wells for high temperatures $(\geq 120 \mathrm{~K})$ under magnetic fields in the Voigt configuration by constructing and numerically solving the kinetic Bloch equations. ${ }^{24,25,26,27,28,34}$ In these studies, we include all the SC scattering such as the electron-phonon, the electron-nonmagnetic impurity as well as the electron-electron Coulomb scattering and investigate the spin dephasing under various conditions. The dephasing obtained from our theory contains both the single-particle dephasing caused by the effective SF scattering first proposed by D'yakonov and Perel', ${ }^{22}$ and the many-body one due to the inhomogeneous broadening provided by the DP term. We show that for the electron densities we studied, the spin dephasing rate is dominated by the many-body effect. Moreover, as we include the electron-electron Coulomb scattering, we are able to investigate the spin dephasing with extra large spin polarization (up to $100 \%$ ) which has not been discussed both theoretically and experimentally. We find that under moderate magnetic fields, the SDT increases dramatically with the initial spin polarization. For example, the SDT of a impurity free sample gets an increase of more than one order of magnitude when the initial spin polarization rises from about 0 to about $100 \%$ at low temperature. ${ }^{33}$ The initial-spinpolarization dependence of the spin dephasing becomes more interesting when the magnetic field is increased to a few tens tesla where the SDT no longer increases monotonically with the initial spin polarization but shows an anomalous resonance peak versus the initial spin polarization. ${ }^{32}$ The dramatic increase and the anomalous resonance of SDT in the high spin polarization region is found to be due to the first order of the electron-electron interaction, i.e., the Hartree-Fock (HF) contribution which provides an effective magnetic field that can reduce the spin dephasing and result in a fast 
increase of the SDT. Moreover, under right condition, the HF term, the applied magnetic field as well as the DP term can reach to a resonance, and thus forms the anomalous peak. Due to the small Landé $g$-factor in GaAs, the resonance condition can only be achieved under very high magnetic fields.

In this paper, we apply the kinetic theory to study the spin dephasing in the $n$-typed InAs QW for high temperatures where the DP term is the leading dephasing mechanism. In QW system, the DP term is composed of the Dresselhaus term ${ }^{35}$ and the Rashba term. ${ }^{36,37}$ The Dresselhaus term is due to the lack of inversion symmetry in the zinc-blende crystal Brillouin zone and is sometimes referred to as bulk inversion asymmetry (BIA) term. Whereas the Rashba term appears if the self-consistent potential within a $\mathrm{QW}$ is asymmetric along the growth direction and is therefore referred to as structure inversion asymmetry (SIA) contribution. For QW's of wide band-gap semiconductors such as GaAs, the Dresselhaus term is the main spin dephasing mechanism. Whereas for QW's composed of narrow band-gap semiconductors such as InAs in the present case, the Rashba term is dominant. As the Rashba term is proportional to the interface electric field of the QW, therefore, the spin dephasing in the InAs QW can be manipulated through applying an electric field perpendicular to the QW. Moreover, as the Landè $g$-factor in InAs is very large $(g=15$ compared to 0.44 of GaAs), one expects to achieve the resonance condition under a moderate magnetic field. We organize the paper as follows: We present our model and the kinetic equations in Sec. II. Then in In Sec. III(A) we investigate how the SDT changes with the variation of the initial spin polarization. The temperature dependence of the SDT under different spin polarization is discussed in detail in Sec. III(B), where we also highlight the difference between the present many-body theory and the earlier simplified theory. In Sec. III(C) we show the magnetic field dependence of the SDT. Finally we discuss how the interface electric field affects the SDT. We present the conclusion and summary in Sec. IV.

\section{KINETIC EQUATIONS}

We start our investigation from an $n$-doped (100) InAs QW with well width $a$. The growth direction is assumed to be $z$-axis. A moderate magnetic field $\mathbf{B}$ is applied along the $x$ axis. Due to the confinement of the QW, the momentum states along $z$ axis are quantized. Therefore the electron states are characterized by a subband index $n$ and a two dimensional wave vector $\mathbf{k}=\left(k_{x}, k_{y}\right)$ together with a spin index $\sigma$. In the present paper, we choose the electron density so that only the lowest subband is populated and the transition to the upper subbands is unimportant. Therefore, one only needs to consider the lowest subband. With the DP term (specifically the Rashba term) included, the Hamiltonian of the electrons in the QW takes the form:

$$
H=\sum_{\mathbf{k} \sigma \sigma^{\prime}}\left\{\varepsilon_{\mathbf{k}}+\left[g \mu_{B} \mathbf{B}+\mathbf{h}(\mathbf{k})\right] \cdot \frac{\vec{\sigma}_{\sigma \sigma^{\prime}}}{2}\right\} c_{\mathbf{k} \sigma}^{\dagger} c_{\mathbf{k} \sigma^{\prime}}+H_{I}
$$

Here $\varepsilon_{\mathbf{k}}=\mathbf{k}^{2} / 2 m^{*}$ is the energy of electron with wavevector $\mathbf{k}$ and effective mass $m^{*}$. $\vec{\sigma}$ are the Pauli matrices. The Rashba term $\mathbf{h}(\mathbf{k})$ can be written as

$$
h_{x}(\mathbf{k})=\alpha k_{y}, h_{y}(\mathbf{k})=-\alpha k_{x}, h_{z}(\mathbf{k})=0 .
$$

In these equations, $\alpha$ is proportional to the interface electric field $E_{z}$ along the growth direction:

$$
\alpha=\alpha_{0} e E_{z},
$$

with the coefficient $\alpha_{0}$ being inversely proportional to the energy gap and the effective mass. ${ }^{38}$ The interaction Hamiltonian $H_{I}$ is composed of Coulomb interaction $H_{e e}$, electron-phonon interaction $H_{p h}$, as well as electronimpurity scattering $H_{i}$. Their expressions can be found in textbooks. ${ }^{39,40}$

We construct the kinetic Bloch equations by the nonequilibrium Green function method ${ }^{39}$ as follows:

$$
\dot{\rho}_{\mathbf{k}, \sigma \sigma^{\prime}}=\left.\dot{\rho}_{\mathbf{k}, \sigma \sigma^{\prime}}\right|_{\mathrm{coh}}+\left.\dot{\rho}_{\mathbf{k}, \sigma \sigma^{\prime}}\right|_{\mathrm{scatt}}
$$

Here $\rho_{\mathbf{k}}$ represents the single particle density matrix. The diagonal elements describe the electron distribution functions $\rho_{\mathbf{k}, \sigma \sigma}=f_{\mathbf{k} \sigma}$. The off-diagonal elements $\rho_{\mathbf{k}, \frac{1}{2}-\frac{1}{2}} \equiv \rho_{\mathbf{k}}$ describe the inter-spin-band polarizations (coherence) of the spin coherence. ${ }^{34}$ Note that $\rho_{\mathbf{k},-\frac{1}{2} \frac{1}{2}} \equiv$ $\rho_{\mathbf{k}, \frac{1}{2}-\frac{1}{2}}^{*}=\rho_{\mathbf{k}}^{*}$. Therefore, $f_{\mathbf{k} \pm \frac{1}{2}}$ and $\rho_{\mathbf{k}}$ are the quantities to be determined from Bloch equations.

The coherent part of the equation of motion for the electron distribution function and the spin coherence are given by

$$
\begin{gathered}
\left.\frac{\partial f_{\mathbf{k}, \sigma}}{\partial t}\right|_{\mathrm{coh}}=-2 \sigma\left\{\left[g \mu_{B} B+h_{x}(\mathbf{k})\right] \operatorname{Im} \rho_{\mathbf{k}}+h_{y}(\mathbf{k}) \operatorname{Re} \rho_{\mathbf{k}}\right\}+4 \sigma \operatorname{Im} \sum_{\mathbf{q}} V_{\mathbf{q}} \rho_{\mathbf{k}+\mathbf{q}}^{*} \rho_{\mathbf{k}}, \\
\left.\frac{\partial \rho_{\mathbf{k}}}{\partial t}\right|_{\text {coh }}=\frac{1}{2}\left[i g \mu_{B} B+i h_{x}(\mathbf{k})+h_{y}(\mathbf{k})\right]\left(f_{\mathbf{k} \frac{1}{2}}-f_{\mathbf{k}-\frac{1}{2}}\right)+i \sum_{\mathbf{q}} V_{\mathbf{q}}\left[\left(f_{\mathbf{k}+\mathbf{q} \frac{1}{2}}-f_{\mathbf{k}+\mathbf{q}-\frac{1}{2}}\right) \rho_{\mathbf{k}}-\rho_{\mathbf{k}+\mathbf{q}}\left(f_{\mathbf{k} \frac{1}{2}}-f_{\mathbf{k}-\frac{1}{2}}\right)\right],
\end{gathered}
$$


respectively, where $V_{\mathbf{q}}=4 \pi e^{2} /\left[\kappa_{0}\left(q+q_{0}\right)\right]$ is the $2 \mathrm{D}$ Coulomb matrix element under static screening. $q_{0}=$ $\left(e^{2} m^{*} / \kappa_{0}\right) \sum_{\sigma} f_{\mathbf{k}=0, \sigma}$ and $\kappa_{0}$ is the static dielectric constant. The first term on the right hand side (RHS) of Eq. (5) describes the spin precession of electrons under the magnetic field $\mathbf{B}$ as well as the effective magnetic field $\mathbf{h}(\mathbf{k})$ due to the Rashba effect. The scattering terms of the electron distribution function and the spin coherence are given by Eqs. (A1) and (A2) in the Appendix.

The initial conditions are taken at $t=0$ as:

$$
\begin{gathered}
\left.\rho_{\mathbf{k}}\right|_{\mathrm{t}=0}=0 \\
\left.f_{\mathbf{k} \sigma}\right|_{\mathrm{t}=0}=1 /\left\{\exp \left[\left(\varepsilon_{\mathbf{k}}-\mu_{\sigma}\right) / k_{B} T\right]+1\right\}
\end{gathered}
$$

where $\mu_{\sigma}$ is the chemical potential for spin $\sigma$. The condition $\mu_{\frac{1}{2}} \neq \mu_{-\frac{1}{2}}$ gives rise to the imbalance of the electron densities of the two spin bands.

\section{NUMERICAL RESULTS}

The kinetic Bloch equations form a set of nonlinear equations. All the unknowns to be solved appear in the scattering terms. Specifically, the electron distribution function is no longer a Fermi distribution because of the existence of the anisotropic Rashba term $\mathbf{h}(\mathbf{k})$. This term in the coherent part drives the electron distribution away from an isotropic Fermi distribution. The scattering term attempts to randomize electrons in k-space. Obviously, both the coherent part and the scattering terms have to be solved self-consistently to obtain the distribution function and the spin coherence.

We numerically solve the kinetic Bloch equations in such a self-consistent fashion to study the spin precession between the spin-up and -down bands. We include electron-phonon scattering and the electron-electron interaction throughout our computation. As we concentrate on the relatively high temperature regime in the present study, for electron-phonon scattering we only need to include electron-LO phonon scattering. Electronimpurity scattering is sometimes excluded. As discussed in the previous paper, ${ }^{34,41}$ irreversible spin dephasing can be well defined by the slope of the envelope of the incoherently summed spin coherence $\rho(t)=\sum_{\mathbf{k}}\left|\rho_{\mathbf{k}}\right|$. The material parameters of InAs for our calculation are tabulated in Table I. ${ }^{42}$ The method of the numerical calculation has been laid out in detail in the previous paper on the DP mechanism in $3 \mathrm{D}$ systems. ${ }^{24}$ The difference is that here we are able to get the results quantitatively in stead of only qualitatively as in the previous $3 \mathrm{D}$ case, thanks to the smaller dimension in the momentum space. Our main results are plotted in Figs. 1 to 6 . In these calculations the total electron density $N_{e}$ and the applied magnetic field $B$ are chose to be $4 \times 10^{10} \mathrm{~cm}^{-2}$ and $1.5 \mathrm{~T}$ respectively unless otherwise specified.

\begin{tabular}{llll}
\hline \hline$\kappa_{\infty}$ & 12.25 & $\kappa_{0}$ & 15.15 \\
$\omega_{0}$ & $27 \mathrm{meV}$ & $m^{*}$ & $0.00239 m_{0}$ \\
$a$ & $7.5 \mathrm{~nm}$ & & \\
\hline \hline
\end{tabular}

TABLE I: Parameters used in the numerical calculations

\section{A. Spin polarization dependence of the spin dephasing time}

We first study the spin polarization dependence of the SDT. As our theory is a many-body theory and we include all the scattering, especially the Coulomb scattering, in our calculation, we are able to calculate the SDT with large spin polarization.

In Fig. 1, SDT $\tau$ is plotted against the initial spin polarization $P$ with $N_{i}=0$ [Fig. $1(\mathrm{a})$ ] and $N_{i}=0.1 N_{e}$ [Fig. 1(b)] at different temperatures. The most striking feature of the impurity-free case is the huge anomalous peaks of the SDT in low temperatures. For $T=120$ $\mathrm{K}$, the peak value of the SDT is about 6 times higher than that of low initial spin polarization. It is also seen from the figure that the anomalous peak is reduced with the increase of temperature and the peak shifts to higher polarization. For $T>200 \mathrm{~K}$ there is no anomalous peak.

The anomalous peak in the $\tau-P$ curve in low temperature region originates from the electron-electron interaction, specifically the Hartree-Fock (HF) self-energy [i.e., the last terms in the Eq. (5) and (6)]. If one removes the $\mathrm{HF}$ term, the anomalous peak as well as the large increase of SDT disappears. It is pointed out in our previous paper that although the HF term itself does not contribute to the spin dephasing directly, ${ }^{25,28}$ it can alert the motion of the electrons as it behaves as an effective magnetic field $\mathbf{B}^{\mathrm{HF}}(\mathbf{k})$. Therefore, the $\mathrm{HF}$ term can affect the spin dephasing by combining with the DP term. For small spin polarization as commonly discussed in the literature, the contribution of the HF term is marginal. However, when the polarization gets higher, the HF contribution becomes larger. Especially the effective magnetic field formed by the HF term contains a longitudinal component $\left[B_{z}^{\mathrm{HF}}(\mathbf{k})\right]$ which can effectively reduce the "detuning" of the spin-up and -down electrons, and thus strongly reduces the spin dephasing, therefore the SDT increases with initial spin polarization. ${ }^{33}$ Moreover, besides the initial polarization, $\rho_{\mathbf{k}}$ and therefore $B^{\mathrm{HF}}(\mathbf{k})$ are also affected by the applied magnetic field. With higher magnetic field, both gets larger. Under the high magnetic field and when the initial spin polarization reaches to a right value, the effective magnetic field $\mathbf{B}^{\mathrm{HF}}(\mathbf{k})$ may reach the magnitude comparable to the contribution from the DP term as well as the applied magnetic field in the coherent parts of the Bloch equations and reduces the anisotropic caused by the DP term. Therefore, one gets much longer SDT. However, if one further increases the initial polarization, the $\mathrm{HF}$ term 

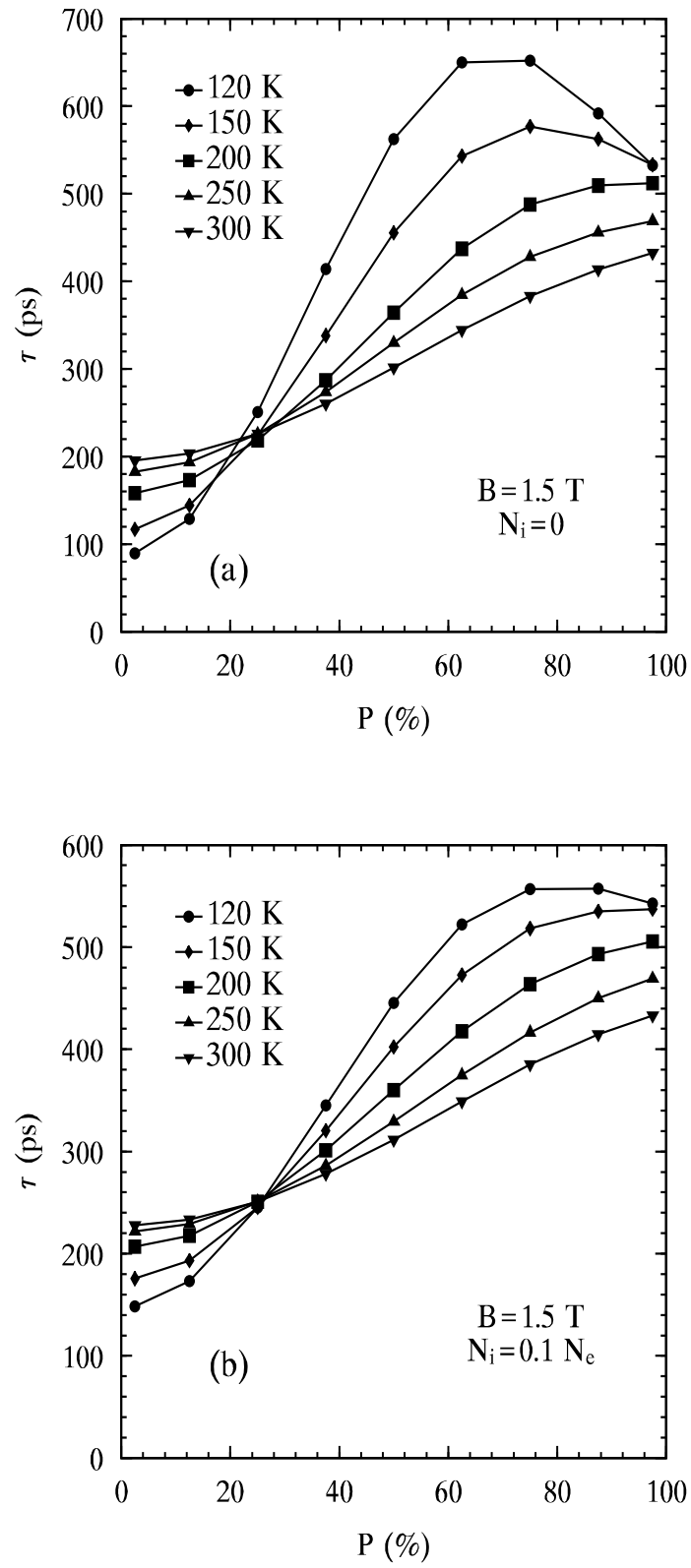

FIG. 1: Spin dephasing time $\tau$ versus the initial spin polarization $P$ with different impurity concentration and different temperatures. The impurity densities in (a) and (b) are 0 and $0.1 N_{e}$ respectively. The lines are plotted for the aid of eyes.

exceeds the resonance condition. As the result, the SDT decreases. Therefore, one gets the anomalous peak which is similar to the resonance effect. It is noted at, as both the DP term and the HF term are $k$-dependent, the resonance is broadened.

For high temperatures the HF term is smaller. In order to reach the resonance, one needs to go to higher polarization. Therefore, as shown in the figure the anomalous peak shifts to the higher polarization. However, when the temperature is high enough, even largest polarization $P=100 \%$ cannot make the HF term to reach the resonance condition. Therefore, the peak disappears.

The $\tau$ - $P$ curve is much different when the impurities are introduced. It is seen from Fig. 1(b) that, when the density of impurity is large, say $N_{i}=0.1 N_{e}$, the fast rise in $\tau$ - $P$ curve remains. Nevertheless the increase is smaller than the corresponding one when the impurities are absent. In addition to the reduction of the rise in $\tau$ - $P$ curves, the impurities destroy the anomaly too. One can easily see that, with the impurity level $N_{i}=0.1 N_{e}$, when the temperature is $120 \mathrm{~K}$, the anomalous peak is much flatter than the impurity free sample, while for all other temperatures we study, the SDT increases uniquely with the polarization.

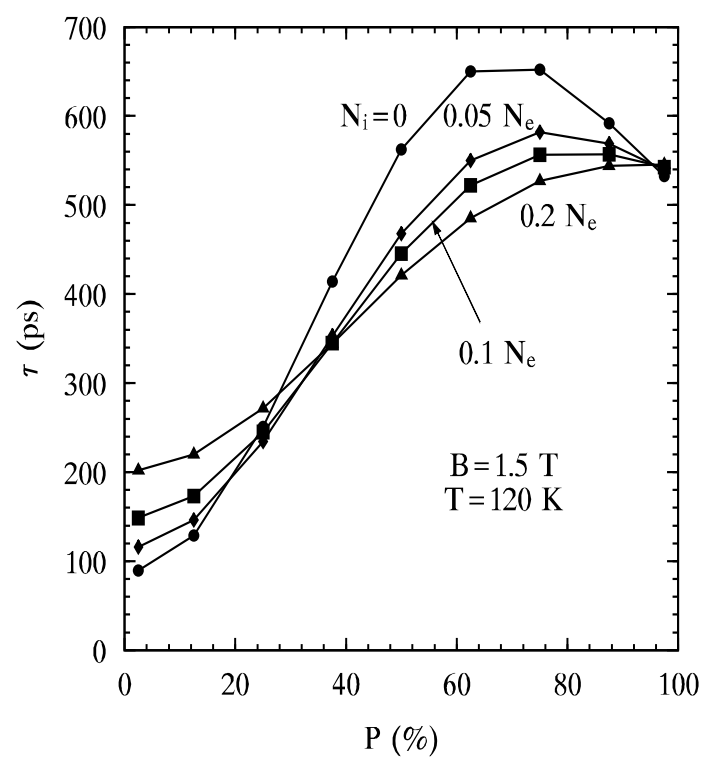

FIG. 2: Spin dephasing time $\tau$ versus the initial spin polarization $P$ for a InAs QW with different impurity levels. Circle $(\bullet): N_{i}=0$; Diamond $(\checkmark): N_{i}=0.05 N_{e} ;$ Square $(\boldsymbol{\square})$ : $N_{i}=0.1 N_{e} ;$ Up triangle $(\boldsymbol{\Lambda}): N_{i}=0.2 N_{e}$. The lines are plotted for the aid of eyes.

To further reveal the contribution of the impurity to the dephasing under different conditions, we plot the SDT as a function of the polarization for different impurity levels at $T=120 \mathrm{~K}$ in Fig. 2. The figure clearly shows that the impurity tends to remove the anomalous peak and to shift the peak to the larger initial spin polarization. This is because that the impurity reduces the $\mathrm{HF}$ term and therefore the resonance effect is also reduced. Hence, in order to reach the maximum resonance, one has to increase the initial spin polarization. Consequently, the peak shifts to larger $P$. Whereas when $N_{i}$ is raised to $0.2 N_{e}$, the HF term is reduced too much to form a peak. 


\section{B. The temperature dependence of the spin dephasing time}

Above we discussed the dependence of spin dephasing on initial spin polarization for different temperatures. Now we turn to the temperature dependence of the SDT under different initial spin polarizations. ¿From Fig. 1(a) and (b) in Sec. III(B), one can see that for small polarization, the SDT increases with the temperature. Whereas in high polarized region, the SDT decreases with the temperature. For moderate polarization, the temperature dependence is too complicated to be described by a monotonic function of temperature.

To see more detail of how the spin dephasing depends on the temperature, we replot the SDT shown in Fig. 1 as a function of the temperature for different impurity levels and different spin polarizations in Fig. 3(a) and (b). It is seen from the figure that similar to the case of GaAs where the DP term is composed of the Dresselhaus term, ${ }^{33}$ for Rashba term and for low spin polarization the SDT also increases with the temperature for all impurity levels. This property is again opposite to the results of earlier simplified treatments of the DP effect, where it was predicted that the spin lifetime decreases with the increase of temperature in the $2 \mathrm{D}$ system. ${ }^{43,44}$ The SDT based on the simplified model is given by ${ }^{27,43,45}$

$$
\frac{1}{\tau}=\frac{\int_{0}^{\infty} d E_{k}\left(f_{k \frac{1}{2}}-f_{k-\frac{1}{2}}\right) \Gamma(k)}{\int_{0}^{\infty} d E_{k}\left(f_{k \frac{1}{2}}-f_{k-\frac{1}{2}}\right)},
$$

in which

$$
\Gamma(k)=2 \tau_{1}(k)\left(\alpha_{0} E k\right)^{2}
$$

and

$$
\tau_{n}^{-1}(k)=\int_{0}^{2 \pi} \sigma\left(E_{k}, \theta\right)[1-\cos (n \theta)] d \theta .
$$

$\sigma\left(E_{k}, \theta\right)$ stands for scattering cross-section. For comparison, we plot the SDT predicated by the earlier model and by our present many-body theory in the inset of Fig. 3(a). ¿From the inset one can see that the SDT predicated by the earlier model is about one order of magnitude larger than the one predicated by our theory. In the mean time, the SDT of the earlier mode drops dramatically with the increase of the temperature. Nevertheless, in our manybody treatment, it rises slightly with the temperature.

The giant difference between two models lies on the fact that the earlier simplified model is based on the single particle picture which does not count for the dephasing due to the inhomogeneous broadening inherited in the Rashba term, which is exactly the result of many body effect. ${ }^{24,25,26,27,28}$ By comparing the theoretical SDT predicated by the two models, we can see that the spin dephasing due to the inhomogeneous broadening is much more important. In the case we calculated, the spin dephasing is dominated by the inhomogeneous broadening. Therefore, it is easy to understand why the earlier simplified treatment of the DP mechanism gives much slower spin dephasing. Although there is no experiment results for InAs, we have shown that for GaAs the prediction of our many-body theory agrees both quantitatively and qualitatively with the experiment results. ${ }^{33}$
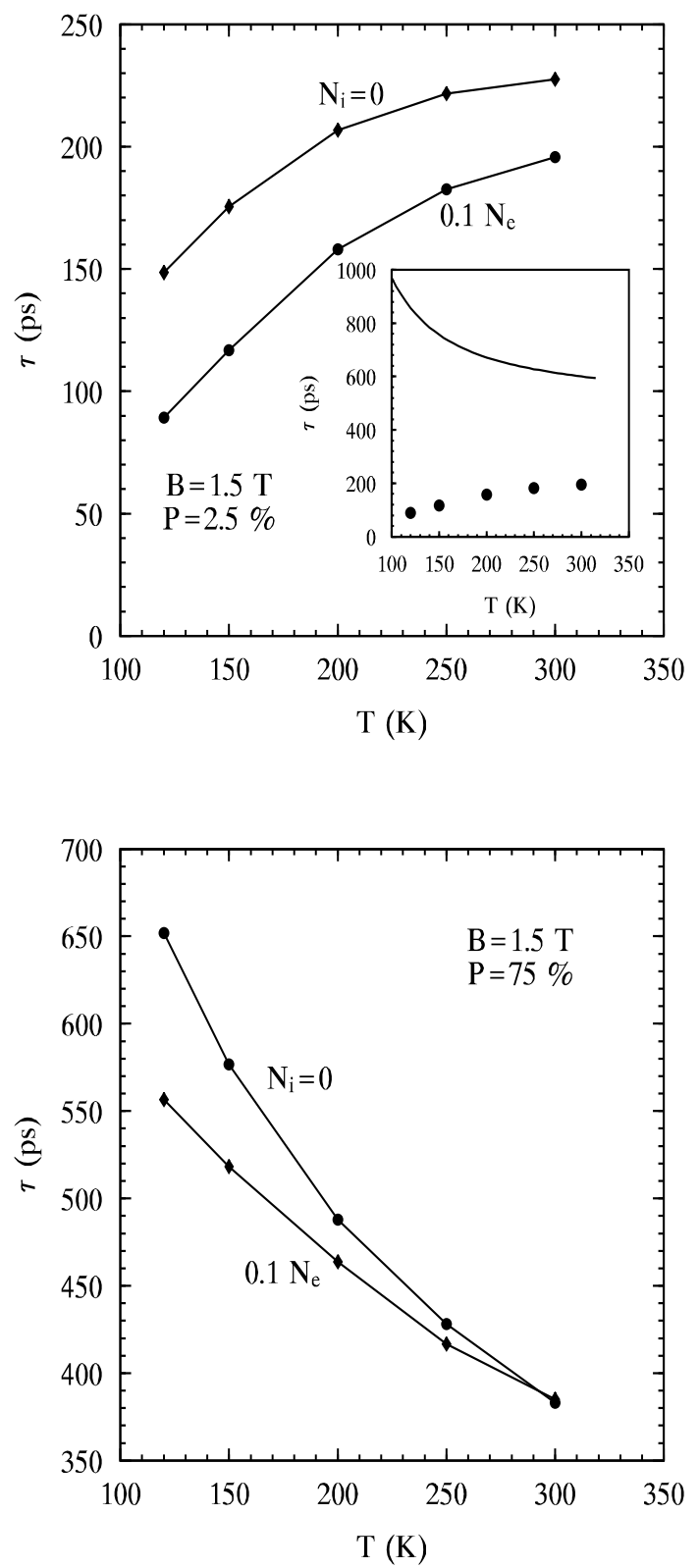

FIG. 3: Spin dephasing time $\tau$ versus the temperature $T$ for InAs QW's with spin polarization $P=2.5 \%$ (a) and $P=$ $75 \%$ (b) under two different impurity levels. Circle $(\bullet): N_{i}=$ 0; Diamond $(\checkmark): N_{i}=0.1 N_{e}$. The lines are plotted for the aid of eyes. The SDT predicated by the simplified treatment of DP term (solid curve) and our model (circle) for $N_{i}=0$ is plotted in the inset of (a) for comparison.

The temperature dependence of the SDT can be easily understood when the spin dephasing due to inhomoge- 
neous broadening is taken into account: When the temperature increases, the inhomogeneous broadening is reduced as the electrons are distributed to the wider $k$ states. As a result, the number of electron occupation on each $\mathbf{k}$ state is reduced. It is further noted that this reduction is mild as function of the temperature. Therefore, the temperature dependence is quit mild unless it is within the regime of anomalous peak.

In the region where HF term is important, in addition to the above mentioned two effects of the temperature acting on the spin dephasing, the temperature dependence of HF term should also be taken into account when we study the temperature dependence of the spin dephasing. In high spin polarization region, the SDT decreases with the temperature. However, in the moderately polarized region, the temperature dependence of SDT due to the combination of these three effects is too complicate to be described by a monotonic function. We replot the SDT as a function of the temperature in Fig. 3(b), for the high polarization $P=75 \%$, which is near the anomalous peak shown in Fig. 1(a). We can see that due to the reduction of the HF term, the resonance is removed and the SDT drops dramatically with the increase of the temperature in the impurities free sample. While for the system with impurity concentration $N_{i}=0.1 N_{e}$, the HF term is less important than that in the impurities free sample, and the SDT is less sensitive to the temperature.

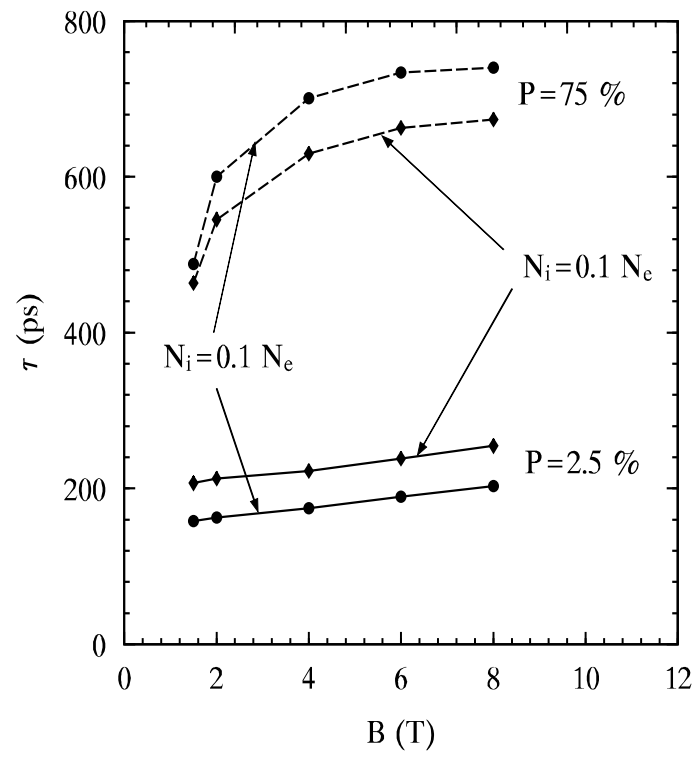

FIG. 4: Spin dephasing time $\tau$ versus the applied magnetic field for InAs QW's for different spin polarizations and different impurity levels. Solid curve with dots: $N_{i}=0, P=2.5 \%$; Solid curve with diamonds: $N_{i}=0.1 N_{e}, P=2.5 \%$; Dashed curve with dots: $N_{i}=0, P=75 \%$; Dashed curve with diamonds: $N_{i}=0.1 N_{e}, P=75 \%$.

\section{Magnetic field dependence of the spin dephasing}

We now investigate the magnetic field dependence of the spin dephasing. In Fig. 4, we plot the SDT versus the applied magnetic field for different impurity levels and different spin polarizations. It is seen that for all the cases we study, the SDT increases with the magnetic field. This is because in the presence of a magnetic field, the electron spins undergo a Larmor precession around the magnetic field. This precession suppresses the precession about the effective magnetic field $\mathbf{h}(\mathbf{k}) .{ }^{18,29}$ Therefore the SDT increases with the magnetic field. It is pointed out that in $3 \mathrm{D}$ electron gas, the magnetic field also forces electrons to precess around it. This precession introduces additional symmetry in the momentum space that limits the $\mathbf{k}$-space available to the DP effect which is anisotropic in it. ${ }^{18,24,29}$ This can further reduce the spin dephasing. However, it is expected that this effect in the $2 \mathrm{D}$ case is less effective than the $3 \mathrm{D}$ case as in $z$-direction the momentum is quantized and the momentum precession around the magnetic field should be suppressed.

In additional to the above mentioned effect of the magnetic field on spin dephasing, one can further see from Fig. 4, that for large polarization, the magnetic field also enhances the HF term. As we mentioned before, for large polarization, the contribution from the HF term is important. Increase of the HF term serves as additional magnetic field which further suppresses the effect of the DP term $\mathbf{h}(\mathbf{k})$, and therefore results in a faster rise in the $\tau$ - $B$ curve in the region of $B<4 \mathrm{~T}$. When the applied magnetic field exceeds $4 \mathrm{~T}$, the increase of HF term saturates, thus the slop of the $\tau-B$ curves in the region of $B>4 \mathrm{~T}$ is reduced to that in the low polarization region. To reveal more concrete about the combining effect of the magnetic field and the HF term on spin dephasing, we plot the SDT as a function of polarization in Fig. 5. It is shown that the rise in the $\tau$ - $P$ curve increases with the magnetic field. Moreover, the position of the peak in $\tau-P$ shifts to a larger polarization. This is understood that, it needs a larger HF term, and hence a larger spin polarization, to achieve the resonance condition when the magnetic field increases. When the magnetic field is raised to $4 \mathrm{~T}$, it is no longer possible to form the resonance for all of the polarization. As a result the SDT increases uniquely with the polarization and there is no peak in the $\tau$-P curve.

\section{Interface electric field dependence of spin dephasing}

We now investigate how the interface electric field affects the spin dephasing. In Fig. 6 we plot the SDT as a function of the initial spin polarization for three different interface electric fields. It can be seen from the figure that when the interface electric field decreases from $2 \times 10^{4} \mathrm{~V} / \mathrm{cm}$ to $5 \times 10^{3} \mathrm{~V} / \mathrm{cm}$, the SDT increases about 


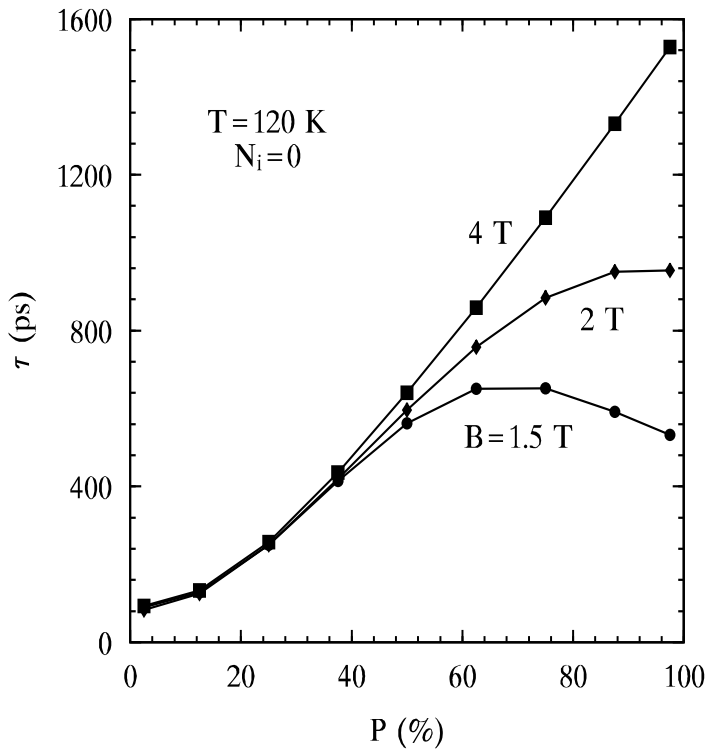

FIG. 5: Spin dephasing time $\tau$ versus the polarization for InAs QW's at different magnetic field. Circle $(\bullet)$ : $B=1.5 \mathrm{~T}$; Diamond $(\downarrow)$ : $B=2 \mathrm{~T}$; Square $(\boldsymbol{\square}): \mathrm{B}=4 \mathrm{~T}$. The lines are plotted for the aid of eyes.

16 folds. It is understood that when the interface electric field increases, the Rashba effect is enhanced, and consequently the spin dephasing is also enhanced. Moreover, for fixed initial spin polarization, the HF term is reduced when $E_{z}$ is increased. As a result, the interface electric field $E_{z}$ also changes the anomalous peak in the $\tau-P$ curve as in order to achieve the resonance condition, one has to go to higher polarization in order to get large enough HF term. Consequently the resonance peak is smoothed and the position moves to higher initial spin polarization region when $E_{z}$ increases, which is shown in the Fig. 6.

\section{CONCLUSION}

In conclusion, we have performed a systematic investigation of the Rashba effect on the spin dephasing of $n$-typed InAs QW's under moderate magnetic fields in Voigt configuration. Based on the nonequilibrium Green's function theory, we derived a set of kinetic Bloch equations for a two-spin-band model. This model includes the electron-phonon, electron-impurity scattering as well as the electron-electron interaction. By numerically solving the kinetic Bloch equations, we study the time evolution of electron densities in each spin band and the spin coherence - the correlation between spin-up and -down bands. The spin dephasing time is calculated from the slope of the envelope of the time evolution of the incoherently summed spin coherence. We therefore

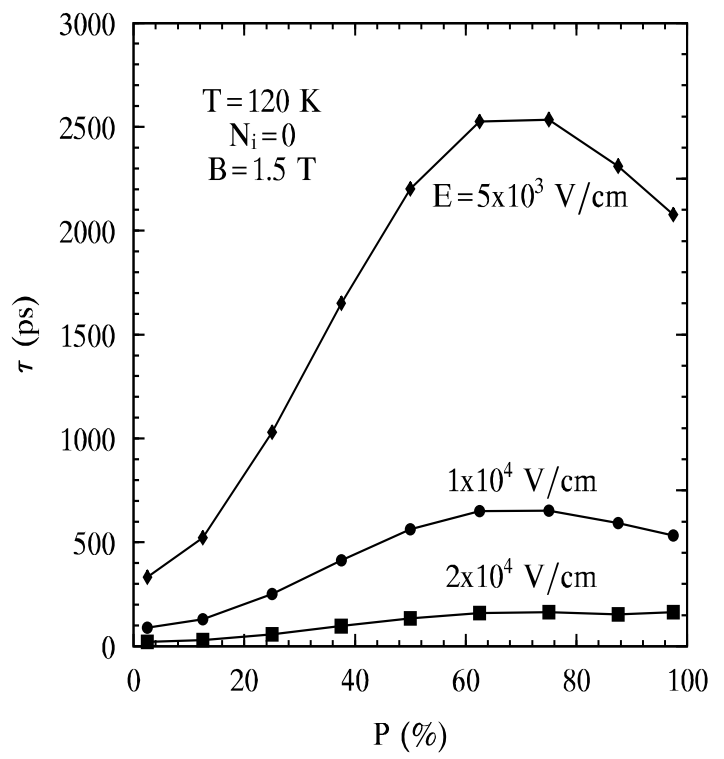

FIG. 6: Spin dephasing time $\tau$ versus the initial spin polarization at $T=120 \mathrm{~K}, B=1.5 \mathrm{~T}, N_{i}=0$ for three different interface electric fields.

are able to study in detail how this dephasing time is affected by spin polarization, temperature, impurity level, magnetic field and interface electric field. Differing from the earlier studies on spin dephasing based on the single particle model which only considers the effective SF scattering, our theory also takes account of the contribution of many-body effect on the spin dephasing. ${ }^{24,25,26,27,28} \mathrm{In}$ fact, for the $n$-typed semiconductors and the spin polarization studied in the experiments, this many-body dephasing effect is even more important than the effective SF scattering as it is one order of magnitude larger than the later. Equally remarkable is that, as we include all the scattering, especially the Coulomb scattering in our many-body theory, now we are able to calculate the spin dephasing with extra large (up to $100 \%$ ) initial spin polarization which is unable to be calculated by the former single particle theory.

It is discovered that the SDT increases with the initial spin polarization. Moreover, for low impurity level and low temperature, there is a giant anomalous resonant peak in the curve of the SDT versus the initial spin polarization. This resonant peak moves to high spin polarization and its magnitude is fast reduced (enhanced) until the whole resonance disappears if one increases the impurity density, the temperature and/or the interface electric field (the magnetic field). It is discovered that this anomalous resonance peak originates from the HF contribution of the electron-electron Coulomb interaction. Under the right spin polarization, the contribution of $\mathrm{HF}$ term may reach the magnitude comparable to the contribution of the DP term as well as the magnetic field in the coherent part of the Bloch equation and reduces the 
anisotropy caused by the Rashba effect - consequently reduces the spin dephasing. As the resonance is the combined effects of the HF term, the Rashba term and the magnetic field, the magnitude and position of the resonance peak are affected by all the factors that can alert the magnitude of the HF term, such as temperature, impurity scattering, magnetic field as well as the interface electric field: For a given impurity concentration, when the temperature increases, the HF term reduces. Consequently the $\tau-P$ curve is smoothed and the peak position is moved to higher spin polarization; For impurity free sample, if the temperature is raised to $200 \mathrm{~K}$, the HF term is reduced too much to form a resonance and the anomalous peak disappears; The same situation happens when the impurity level increases at a given temperature as the scattering also lowers the HF term. When the impurity level is raised to $0.2 N_{e}$ there is no resonance in the temperature region we studied; While the increase of the magnetic field enhances the HF term and results in a faster increase of the SDT as well as a higher resonant peak in $\tau-P$ curve. Moreover, as the magnetic field becomes larger, it needs a larger HF term and hence a larger polarization in order to achieve the resonant condition. Therefore the peak position is also moved to higher polarization; When the interface electric field increases, the HF term is reduced. Therefore the resonance peak in $\tau$-P becomes flatter and its position moves to higher spin polarization.

For low spin polarized regime, the SDT increases when the temperature rises. This is contrary to the result of earlier simplified single-particle calculation where the SDT always decreases with the increase of the temperature. Moreover, the SDT predicted by our many-body calculation is one order of magnitude faster than the earlier result. The physics of this feature is due to the additional many-body spin dephasing channel due to the inhomogeneous broadening provided by the Rashba term, which by combining with the SC scattering also causes spin dephasing. In the situation we studied, the spin dephasing is dominated by the many-body dephasing effect. With the increase of the temperature, the inhomogeneous broadening reduces and the SDT increases.

In high spin polarization region, the contribution of the HF term should be taken into consideration. This brings more complication in the study of the spin dephasing. Usually the SDT can not be described by a monotonic function of the temperature and the impurity concentration in high polarization regime. For polarization near the resonance peak in low temperature and impurities free samples, the SDT decreases dramatically with the temperature as the resonance is removed when the temperature increases. Whereas when the impurity concentration is $0.1 N_{e}$, the SDT is less sensitive to the temperature.

As the magnetic field causes the electron spins to precess about it, this precession will suppress the precession about the effective magnetic field $\mathbf{h}(\mathbf{k})$ originated from the Rashba effect. As a result the spin dephasing is reduced. The magnetic field also enhances the HF term, which servers as an additional magnetic field and further suppresses the Rashba effect. Therefore, the $\tau$-B curve gets a faster increase in the high polarization region. Our calculation also shows that when the interface electric field increases, the SDT decreases. This is because with the increasing of the interface electric field, the Rashba term is strengthened.

In summary we have performed a thorough investigation of the spin dephasing in $n$-typed InAs QW's. Many new features which have not been investigated both theoretically and experimentally are predicted in a wide range of parameters.

\section{Acknowledgments}

MWW is supported by the "100 Person Project" of Chinese Academy of Sciences and Natural Science Foundation of China under Grant No. 10247002. He would also like to thank S. T. Chui at Bartol Research Institute, University of Delaware for hospitality.

\section{APPENDIX A: APPENDIXES}

The scattering terms of electron distribution functions in the Markovian limit are given by

$$
\begin{aligned}
\left.\frac{\partial f_{\mathbf{k}, \sigma}}{\partial t}\right|_{\text {scatt }}= & \left\{-2 \pi \sum_{\mathbf{q} q_{z} \lambda} g_{\mathbf{Q} \lambda}^{2} \delta\left(\varepsilon_{\mathbf{k}}-\varepsilon_{\mathbf{k}-\mathbf{q}}-\Omega_{\mathbf{q} q_{z} \lambda}\right)\left[N_{\mathbf{q} q_{z} \lambda}\left(f_{\mathbf{k} \sigma}-f_{\mathbf{k}-\mathbf{q} \sigma}\right)+f_{\mathbf{k} \sigma}\left(1-f_{\mathbf{k}-\mathbf{q} \sigma}\right)-\operatorname{Re}\left(\rho_{\mathbf{k}} \rho_{\mathbf{k}-\mathbf{q}}^{*}\right)\right]\right. \\
& -2 \pi N_{i} \sum_{\mathbf{q}} U_{\mathbf{q}}^{2} \delta\left(\varepsilon_{\mathbf{k}}-\varepsilon_{\mathbf{k}-\mathbf{q}}\right)\left[f_{\mathbf{k} \sigma}\left(1-f_{\mathbf{k}-\mathbf{q} \sigma}\right)-\operatorname{Re}\left(\rho_{\mathbf{k}} \rho_{\mathbf{k}-\mathbf{q}}^{*}\right)\right]-2 \pi \sum_{\mathbf{q} \mathbf{k}^{\prime} \sigma^{\prime}} V_{\mathbf{q}}^{2} \delta\left(\varepsilon_{\mathbf{k}-\mathbf{q}}-\varepsilon_{\mathbf{k}}+\varepsilon_{\mathbf{k}^{\prime}}-\varepsilon_{\mathbf{k}^{\prime}-\mathbf{q}}\right) \\
& {\left.\left[\left(1-f_{\mathbf{k}-\mathbf{q} \sigma}\right) f_{\mathbf{k} \sigma}\left(1-f_{\mathbf{k}^{\prime} \sigma^{\prime}}\right) f_{\mathbf{k}^{\prime}-\mathbf{q} \sigma^{\prime}}+\frac{1}{2} \rho_{\mathbf{k}} \rho_{\mathbf{k}-\mathbf{q}}^{*}\left(f_{\mathbf{k}^{\prime} \sigma^{\prime}}-f_{\mathbf{k}^{\prime}-\mathbf{q} \sigma^{\prime}}\right)+\frac{1}{2} \rho_{\mathbf{k}^{\prime}} \rho_{\mathbf{k}^{\prime}-\mathbf{q}}^{*}\left(f_{\mathbf{k}-\mathbf{q} \sigma}-f_{\mathbf{k} \sigma}\right)\right]\right\} } \\
& -\left\{\mathbf{k} \leftrightarrow \mathbf{k}-\mathbf{q}, \mathbf{k}^{\prime} \leftrightarrow \mathbf{k}^{\prime}-\mathbf{q}\right\},
\end{aligned}
$$

in which $\left\{\mathbf{k} \leftrightarrow \mathbf{k}-\mathbf{q}, \mathbf{k}^{\prime} \leftrightarrow \mathbf{k}^{\prime}-\mathbf{q}\right\}$ stands for the same terms as in the previous \{\} but with the interchange $\mathbf{k} \leftrightarrow \mathbf{k}-\mathbf{q}$ and $\mathbf{k}^{\prime} \leftrightarrow \mathbf{k}^{\prime}-\mathbf{q}$. The first term inside the braces on the RHS of Eq. (A1) comes from the electron-phonon interaction. 
$\lambda$ stands for the different phonon modes, i.e., one longitude optical (LO) phonon mode, one longitudinal acoustic (AC) phonon mode due to the deformation potential, and two AC modes due to the transverse piezoelectric field. $g_{\mathbf{q} q_{z} \lambda}$ are the matrix elements of electron-phonon coupling for mode $\lambda$. For LO phonons, $g_{\mathbf{q} q_{z} \mathrm{LO}}^{2}=\left\{4 \pi \alpha \Omega_{\mathrm{LO}}^{3 / 2} /\left[\sqrt{2 \mu}\left(q^{2}+\right.\right.\right.$ $\left.\left.\left.q_{z}^{2}\right)\right]\right\}\left|I\left(i q_{z}\right)\right|^{2}$ with $\alpha=e^{2} \sqrt{\mu /\left(2 \Omega_{\mathrm{LO}}\right)}\left(\kappa_{\infty}^{-1}-\kappa_{0}^{-1}\right) . \kappa_{\infty}$ is the optical dielectric constant and $\Omega_{\mathrm{LO}}$ is the LO phonon frequency. The form factor $\left|I\left(i q_{z}\right)\right|^{2}=\pi^{2} \sin ^{2} y /\left[y^{2}\left(y^{2}-\pi^{2}\right)^{2}\right]$ with $y=q_{z} a / 2 . N_{\mathbf{q} q_{z} \lambda}=1 /\left[\exp \left(\Omega_{\mathbf{q} q_{z} \lambda} / k_{B} T\right)-1\right]$ is the Bose distribution of phonon mode $\lambda$ at temperature $T$. The second term inside the braces on the RHS of Eq. (A1) results from the electron-impurity scattering under the random phase approximation with $N_{i}$ denoting the impurity concentration. $U_{\mathbf{q}}^{2}=\sum_{q_{z}}\left\{4 \pi Z_{i} e^{2} /\left[\kappa_{0}\left(q^{2}+q_{z}^{2}\right)\right]\right\}^{2}\left|I\left(i q_{z}\right)\right|^{2}$ is the electron-impurity interaction matrix element with $Z_{i}$ stands for the charge number of the impurity. $Z_{i}$ is assumed to be 1 throughout our calculation. The third term is the contribution of the Coulomb interaction. Similarly, the scattering parts of the spin coherence are given by

$$
\begin{aligned}
\left.\frac{\partial \rho_{\mathbf{k}}}{\partial t}\right|_{\text {scatt }}= & \left\{\pi \sum_{\mathbf{q} q_{z} \lambda} g_{\mathbf{q} q_{z} \lambda}^{2} \delta\left(\varepsilon_{\mathbf{k}}-\varepsilon_{\mathbf{k}-\mathbf{q}}-\Omega_{\mathbf{q} q_{z} \lambda}\right)\left[\rho_{\mathbf{k}-\mathbf{q}}\left(f_{\mathbf{k} \frac{1}{2}}+f_{\mathbf{k}-\frac{1}{2}}\right)+\left(f_{\mathbf{k}-\mathbf{q} \frac{1}{2}}+f_{\mathbf{k}-\mathbf{q}-\frac{1}{2}}-2\right) \rho_{\mathbf{k}}-2 N_{\mathbf{q} q_{z} \lambda}\left(\rho_{\mathbf{k}}-\rho_{\mathbf{k}-\mathbf{q}}\right)\right]\right. \\
& +\pi N_{i} \sum_{\mathbf{q}} U_{\mathbf{q}}^{2} \delta\left(\varepsilon_{\mathbf{k}}-\varepsilon_{\mathbf{k}-\mathbf{q}}\right)\left[\left(f_{\mathbf{k} \frac{1}{2}}+f_{\mathbf{k}-\frac{1}{2}}\right) \rho_{\mathbf{k}-\mathbf{q}}-\left(2-f_{\mathbf{k}-\mathbf{q} \frac{1}{2}}-f_{\mathbf{k}-\mathbf{q}-\frac{1}{2}}\right) \rho_{\mathbf{k}}\right] \\
& -\sum_{\mathbf{q} \mathbf{k}^{\prime}} \pi V_{\mathbf{q}}^{2} \delta\left(\varepsilon_{\mathbf{k}-\mathbf{q}}-\varepsilon_{\mathbf{k}}+\varepsilon_{\mathbf{k}^{\prime}}-\varepsilon_{\mathbf{k}^{\prime}-\mathbf{q}}\right)\left(\left(f_{\mathbf{k}-\mathbf{q} \frac{1}{2}} \rho_{\mathbf{k}}+\rho_{\mathbf{k}-\mathbf{q}} f_{\mathbf{k}-\frac{1}{2}}\right)\left(f_{\mathbf{k}^{\prime} \frac{1}{2}}-f_{\mathbf{k}^{\prime}-\mathbf{q} \frac{1}{2}}+f_{\mathbf{k}^{\prime}-\frac{1}{2}}-f_{\mathbf{k}^{\prime}-\mathbf{q}-\frac{1}{2}}\right)\right. \\
& +\rho_{\mathbf{k}}\left[\left(1-f_{\mathbf{k}^{\prime} \frac{1}{2}}\right) f_{\mathbf{k}-\mathbf{q} \frac{1}{2}}+\left(1-f_{\mathbf{k}^{\prime}-\frac{1}{2}}\right) f_{\mathbf{k}-\mathbf{q}-\frac{1}{2}}-2 \operatorname{Re}\left(\rho_{\mathbf{k}^{\prime}}^{*} \rho_{\mathbf{k}-\mathbf{q}}\right)\right]-\rho_{\mathbf{k}-\mathbf{q}}\left[f_{\mathbf{k}^{\prime} \frac{1}{2}}\left(1-f_{\mathbf{k}^{\prime}-\mathbf{q} \frac{1}{2}}\right)\right. \\
& \left.\left.\left.+\left(1-f_{\mathbf{k}^{\prime}-\frac{1}{2}}\right) f_{\mathbf{k}^{\prime}-\mathbf{q}-\frac{1}{2}}-2 \operatorname{Re}\left(\rho_{\mathbf{k}^{\prime}}^{*} \rho_{\mathbf{k}^{\prime}-\mathbf{q}}\right)\right]\right)\right\}-\left\{\mathbf{k} \leftrightarrow \mathbf{k}-\mathbf{q}, \mathbf{k}^{\prime} \leftrightarrow \mathbf{k}^{\prime}-\mathbf{q}\right\} .
\end{aligned}
$$

* Author to whom correspondence should be addressed; Electronic address: mwwu@ustc.edu.cn

$\dagger$ Mailing Address.

1 M. Ziese and M. J. Thornton, Spin Electronics (Springer, Berlin, 2001)

2 S. A. Wolf, D. D. Awschalom, R. A. Buhrmann, J. M. Daughton, S. von Malnàr, M. L. Roukes, A. Y. Chtchelkanova, and D. M. Treger, Science 294, 1488 (2001).

3 D. D. Awschalom, N. Samarth, and D. Loss, Semiconductor Spintronics and Quantum Computation (Springer, Berlin, 2002).

4 T. C. Damen, L. Vina, J. E. Cunningham, J. Shah, and L. J. Sham, Phys. Rev. Lett. 67, 3432 (1991).

5 J. Wagner, H. Schneider, D. Richards, A. Fischer, and K. Ploog, Phys. Rev. B 47, 4786 (1993).

6 J. J. Baumberg, S. A. Crooker, D. D. Awschalom, N. Samarth, H. Luo, and J. K. Furdyna, Phys. Rev. Lett. 72, 717 (1994).

7 J. J. Baumberg, S. A. Crooker, D. D. Awschalom, N. Samarth, H. Luo, and J. K. Furdyna, Phys. Rev. B 50, 7689 (1994).

8 A. P. Heberle, W. W. Rühle, and K. Ploog, Phys. Rev. Lett. 72, 3887 (1994).

9 C. Buss, R. Frey, C. Flyizanis, and J. Cibert, Solid State Commun. 94, 543 (1995).

10 S. A. Crooker, J. J. Baumberg, F. Flack, N. Samarth, and D. D. Awschalom, Phys. Rev. Lett. 77, 2814 (1996).

11 S. A. Crooker, D. D. Awschalom, J. J. Baumberg, F. Flack, and N. Samarth, Phys. Rev. B. 56, 7574 (1996).

12 C. Buss, R. Pankoke, P. Leisching, J. Cibert, R. Frey, and C. Flytzanis, Phys. Rev. Lett. 78, 4123 (1997).
13 J. M. Kikkawa, I. P. Smorchkova, N. Samarth, and D. D. Awschalom, Science 277, 1284 (1997).

14 J. M. Kikkawa and D. D. Awschalom, Nature 397, 139 (1998).

15 J. Kikkawa and D. Awschalom, Phys. Rev. Lett. 80, 4313 (1998).

16 H. Ohno, Science 281, 951 (1998).

17 Y. Ohno, R. Terauchi, T. Adachi, F. Matsukura, and H. Ohno, Phys. Rev. Lett. 83, 4196 (1999).

18 F. Meier and B. P. Z. (Eds), Optical Orientation (NorthHolland, Amsterdam, 1984).

19 A. G. Aronov, G. E. Pikus, and A. N. Titkov, Zh. Eksp. Teor. Fiz. 84, 1170 (1983), [Sov. Phys.-JETP 57, 680 (1983)].

20 Y. Yafet, Phys. Rev. 85, 478 (1952).

21 R. J. Elliot, Phys. Rev. 96, 266 (1954).

22 M. I. D'yakonov and V. I. Perel', Zh. Eksp. Teor. Fiz. 60, 1954 (1971), [Sov. Phys.-JETP 38, 1053 (1971)].

23 G. L. Bir, A. G. Aronov, and G. E. Pikus, Zh. Eksp. Teor. Fiz. 69, 1382 (1975), [Sov. Phys.-JETP 42, 705 (1975)].

24 M. W. Wu and C. Z. Ning, phys. stat. sol. B 222, 523 (2000).

25 M. W. Wu, J. Supercond.: Incorping Novel Mechanism 14, 245 (2001), cond-mat/0109258.

26 M. W. Wu and M. Kuwata-Gonokami, Solid State Commun. 121, 509 (2002).

27 M. W. Wu, J. Phys. Soc. Jap. 70, 2195 (2001).

28 M. W. Wu and C. Z. Ning, Eur. Phys. J. B. 18, 373 (2000).

29 F. X. Bronold, I. Martin, A. Saxena, and D. L. Smith, Phys. Rev. B 66, 233206 (2002).

${ }^{30}$ M. Q. Weng and M. W. Wu, Phys. Rev. B 66, 235109 
(2002).

31 M. Q. Weng and M. W. Wu, J. Appl. Phys. 93, 410 (2003).

32 M. Q. Weng and M. W. Wu, cond-mat $/ 0210313$.

33 M. Q. Weng and M. W. Wu, cond-mat/0302330.

34 M. W. Wu and H. Metiu, Phys. Rev. B 61, 2945 (2000).

${ }^{35}$ G. Dresselhaus, Phys. Rev. 100, 580 (1955).

36 Y. A. Bychkov and E. Rashba, J. Phys. C 17, 6039 (1984).

37 Y. A. Bychkov and E. Rashba, Sov. Phys. JETP Lett. 39, 78 (1984).

38 G. Lommer, F. Malcher, and U. Rössler, Phys. Rev. Lett. 60, 728 (1988).

39 Haug and A. P. Jauho, Quantum Kinetics in Transport and Optics of Semiconductors (Springer-Verlag, Berlin, 1996).

40 G. D. Mahan, Many-particle Physics (Plenum, New York,
1981).

41 T. Kuhn and F. Rossi, Phys. Rev. Lett. 69, 977 (1992).

42 O. Madelung, M. Schultz, and H. W. (eds.), Numerical Data and Functional Relationships in Science and Technology, Landolt-Börnstein, New Series, vol. 17 (SpringerVerlag, Berlin, 1982).

43 N. S. Averkiev, L. E. Golub, and M. Willander, J. Phys.: Cond. Mat. 14, R271 (2002).

44 M. I. Dyakonov and V. Y. Kachorovskii, Fiz. Tekh. Poluprovodn. 20, 178 (1986), [Soviet Phys. - Semicond. 20, 110 (1986)].

45 W. H. Lau, J. T. Oleberg, and M. E. Flatté, Phys. Rev. B 64, 161301 (2001). 